\title{
The scopes and falls of molecular targeted therapy in gastric cancer
}

\author{
Vinod Vijay Subhash ${ }^{1}$ and Wei Peng Yong ${ }^{1,2 *}$ \\ ${ }^{1}$ Cancer Science Institute of Singapore, Yong loo Lin School of Medicine, National University of Singapore, Singapore \\ ${ }^{2}$ Department of Haematology-Oncology, National University Hospital, Singapore
}

\begin{abstract}
Genome expression profiling in gastric cancer patients have shown unique molecular phenotypes that may cause differences in the pathological appearance and clinical behaviour of tumor. Interestingly, multiple alterations observed in the expression of receptor tyrosine kinases in the gastric cancer genome reveal multiple pathways that could be targeted therapeutically. Thus manipulation of these pathways using molecular targeting agents presents us with strategies that could change the existing treatment paradigms in gastric cancer therapy. Recent advances in molecular targeted therapies have shown huge prospects, however requires deeper insights into the tumor biology and selection of patient subtypes. In this review, we will discuss the current strategies, advances and prospects of molecular targeted therapies in gastric cancer. Special focus is being laid on Her2, EGFR, VEGF targeting and clinical trials that attempt to further enhance the treatment outcomes and clinical management of gastric cancer patients.
\end{abstract}

\section{Introduction}

Gastric cancer is the second leading cause of cancer-related deaths worldwide [1]. The disease is generally asymptomatic and is diagnosed often at late stage, resulting in metastasis of cancer that can progress to an advanced and even terminal stage. Surgical resection remains the mainstay of curative-intend treatment in early gastric cancer [2]. In an advanced stage, treatment is largely palliative and consists of chemotherapy and radiation. The combination of a fluorinated pyrimidine with a platinum agent have improved therapy convenience and lessened toxicity. However, the median survival of patients with metastatic disease remains between 6 and 9 months. The second line treatment using taxanes and irinotecan also show modest survival benefits and treatment tolerance. Gastric cancer is a heterogeneous disease which may be divided into subgroups based on histological, anatomical, epidemiological and molecular classifications. Distinct molecular drivers and tumor biology, and thus different treatment targets and predictive biomarkers, may be implicated in each subtype [3]. The definition of cell surface antigens that are expressed by human cancers has revealed a broad array of targets that are over expressed, mutated or selectively expressed compared with normal tissues. A key challenge has been to identify antigens that are suitable for antibodybased therapeutics [4]. Monoclonal antibodies are most successful in immunotherapy clinically. They mediate antitumor activity via antibody-dependent cell-mediated cytotoxicity (ADCC), phagocytosis and complement-mediated cytotoxicity [5]. The advantages of monoclonal antibodies in cancer immunotherapy include specific targeting of tumor cell while sparing normal tissue, relative ease of administration and low toxicity profile. On the other hand, major disadvantages include absence of direct $\mathrm{T}$ cell activation and generation of memory immune cells. Additionally, a potential limiting factor in the use of mAbs involves tumor heterogeneity [6]. Here we review multiple strategies and associated clinical trials that explore the prospects of antibody based targeted therapy in gastric cancer.

\section{Antibodies targeting tumor antigens}

Antigens that are involved in growth and differentiation signaling are often growth factors and growth factor receptors. One group of representative targets are the oncogenenic receptor tyrosine kinases (RTKs) that play an important role in the control of much fundamental process, such as growth, differentiation, adhesion, migration and apoptosis [7-9]. Several RTKs are activated in advanced gastric cancer and various RTK inhibitors have been developed as tailored therapy [10]. The results of recent clinical trials in gastric cancer patients evaluate the effectiveness of targeting RTKs (Table 1).

\section{Her2 inhibition}

The tyrosine kinase receptor HER-2/neu is the target structure for the humanized $\mathrm{mAb}$ trastuzumab which has been successfully used for the treatment of breast and gastric cancer [10]. HER-2 over expression is observed in $10-38 \%$ of gastric cancer patients [11,12]; Recently, Bang et al. [13] reported that HER-2-positive patients using Immunohistochemistry (IHC) scoring system had a superior outcome when treated with conventional chemotherapy with trastuzumab, which selectively binds to HER-2 and inhibits its downstream signaling pathway, in the ToGA trial. The ToGA trial had a total of 594 patients with gastric or gastroesophageal junction cancer that overexpressed HER-2 protein (as determined by immunohistochemistry or gene amplification by fluorescence in situ hybridization) were randomly assigned to the study treatments (trastuzumab plus chemotherapy,

Correspondence to: Wei Peng Yong, Department of Haematology-Oncology, National University Cancer Institute, Singapore; National University Hospital of Singapore, Level 7, NUHS Tower Block, 1E, Kent Ridge Road, Singapore 119228, Tel: +65 9654 0060, Fax: +65 6872 3137; E-mail: wei_peng_yong@nuhs.edu.sg

Key words: gastric cancer, molecular targeting, Her2, EGFR, VEGF

Received: July 17, 2015; Accepted: August 17, 2015; Published: August 20, 2015 
Table 1. Phase III trials of targeted Receptor tyrosine kinases in advanced gastric cancer.

\begin{tabular}{|l|c|c|c|c|}
\hline Reference & Clinical trial & Setting & RTK inhibitor & Chemotherapeutic drug \\
\hline NCT01041404 & ToGA & Ist line & Trastuzumab & FP or XP \\
\hline NCT00548548 & AVAGAST & Ist line & Bevacizumab & XP \\
\hline NCT00678535 & EXPAND & Ist line & Cetuximab & Completed \\
\hline NCT00824785 & REAL-3 & Ist line & Panitumumab & Completed \\
\hline NCT00486954 & LoGIG & Ist line & Lapatinib & T \\
\hline NCT00486954 & TYTAN & IInd line & Rapatinib & Completed \\
\hline NCT00917384 & REGARD & Ind line & Ramucirumab & Completed \\
\hline NCT01170663 & RAINBOW & IInd line & Onartuzumab & Tompleted \\
\hline NCT01662869 & metMAB & Ist line & FLO \\
\hline
\end{tabular}

ToGA: Trastuzumab for Gastric Cancer, AVAGAST: Avastin in Gastric Cancer, EXPAND: Erbitux in combination with Xeloda and Cisplatin in Advanced Esophago-Gastric Cancer, REAL-3: Randomized ECF for Advanced and Locally Advanced Esophagogastric Cancer 3, LoGIG: Lapatinib Optimization Study in ErbB2 (HER-2) Positive Gastric Cancer, TYTAN: Lapatinib (Tykerb) with Paclitaxel (Taxol) in Asian ErbB2+ (HER+) Gastric Cancer Study, RAINBOW: Ramucirumab (IMC-1211B) in Metastatic Gastric Adenocarcinoma, metMAB F: 5-Fluorouracil, P: Cisplatin, X: Capecitabine, E: Epirubicin, T: Paclitaxel. O: Oxaliplatin, L: Leucovorin, BSC: Best Supportive Care

$n=298$; chemotherapy alone, $n=296$ ); of these patients, 584 were included in the primary analysis ( $n=294$ and $n=290$, respectively). The median overall survival in the trastuzumab plus chemotherapy arm was 13.8 mo (95\%CI: 12-16) compared with 11.1 mo (95\%CI: 10-13) in the chemotherapy alone arm (HR=0.74, 95\%CI: $0.60-0.91, P=0.0046)$. The study met not only the primary endpoint of improved overall survival but also the secondary endpoint of improved response rates and progression-free survival.

\section{EGFR inhibition}

EGFR is over expressed in $27-64 \%$ of gastric cancers, especially in the more proximal tumors [14]. Cetuximab (Erbitux, Imclone Systems) is a recombinant humanized murine monoclonal antibody against EGFR and is the most investigated anti-EGFR therapy in gastric cancer. The results of the large, randomized, phase III EXPAND study (NCT00678535), which investigated the addition of cetuximab to doublet chemotherapy showed no clinically significant benefit. The median progression-free survival (PFS) for the 455 patients administered the capecitabine-cisplatin plus cetuximab treatment was $4.4 \mathrm{mo}$ (95\%CI: 4.2-5.5) compared to $5.6 \mathrm{mo}$ (95\%CI: 5.1-5.7) for the 449 patients treated with capecitabine-cisplatin alone $(\mathrm{HR}=1.09$, 95\%CI: 0.92-1.29; $P=0.32$ ). Another mono clonal antibody that target EGFR is Panitumumab Van Cutsem et al. [15] reported a phase III trial of panitumumab plus best supportive care compared to best supportive care alone in patients with advanced colorectal cancer that failed to respond to 5-FU, irinotecan and oxaliplatin. However, there are very few reports of this agent being used to treat AGC patients. The results of a randomized, open-label, phase III trial for patients with previously untreated advanced esophagogastric cancer (REAL3) were revealed; this study examined two groups of esophagogastric cancer patients treated with epirubicin, oxaliplatin and capecitabine with or without panitumumab [16]. The median overall survival of the 275 patients with advanced esophagogastric adenocarcinoma in the epirubicin, oxaliplatin and capecitabine (EOC) treatment group was 11.3 mo (95\%CI: 9.6-13.0) compared to 8.8 mo (95\%CI: 7.7-9.8) in the 278 patients treated with modified-dose EOC plus panitumumab $(\mathrm{mEOC}+\mathrm{P})(\mathrm{HR}=1.37,95 \% \mathrm{CI}: 1.07-1.76 ; P=0.013)$.

\section{VEGF inhibition}

VEGF over expression in gastric cancer is associated with hematogenous metastasis, peritoneal dissemination and poor prognosis [17]. The phase III Avastin in Gastric Cancer (AVAGAST) trial was designed to evaluate the efficacy of adding bevacizumab to first-line capecitabine-cisplatin treatment for advanced gastric cancer. Bevacizumab binds to vascular endothelial growth factor (VEGF) and inhibit VEGFR signalling. In total, 774 patients were randomly separated and administered capecitabine and cisplatin with or without bevacizumab. The ORR significantly improved with the addition of bevacizumab ( $46 \%$ vs $37 \%$; $P=0.0315$ ), and the median PFS was also significantly longer (6.7 vs $5.3 \mathrm{mo}$; $\mathrm{HR}=0.80$; $95 \% \mathrm{CI}$ : $0.68-0.93$; $P=0.0037)$. The clinical outcomes were different depending on the geographical region. Survival was extended in Pan-American patients who were treated with bevacizumab; however, this was not the case for Asians or Europeans, despite the better prognosis of the latter. Differences in population genetics, patient selection and second-line chemotherapy may explain these results. Ramucirumab is another VEGFR enhibitor drug that binds with high affinity to the extracellular VEGF-binding domain of VEGFR-2. The phase III RAINBOW trial is a randomized multicenter double-blind, placebo controlled trial evaluating the safety and efficacy of paclitaxel plus ramucirumab drug product compared to paclitaxel plus placebo [18]. The international phase III REGARD study demonstrated improved overall survival with ramucirumab as second-line therapy for patients with advancedstage gastric and gastroesophageal junction adenocarcinoma [19]. Ramucirumab has also received CHMP recommendation in Europe based on positive clinical efficacy data from the REGARD trial as the first targeted agent for the second-line treatment setting in gastric cancer

\section{Other targeted agents}

c-Met is a proto-oncogene that encodes a protein known as hepatocyte growth factor receptor (HGFR). A high level of c-Met expression has been correlated with poor survival in patients with gastric cancer [20]. c-Met inhibitors include monoclonal antibodies and small molecules that inhibit the enzymatic activity of the c-Met TK. Onartuzumab is a humanized mAb directed against HGFR. A randomized, phase III, multicenter, doubleblind, placebo-controlled study evaluating the efficacy and safety of onartuzumab in combination with mFOLFOX6 in patients with metastatic Her2-negative, c-Metpositive gastroesophageal cancer is now ongoing. Other miltitargeted drugs which are in early stages of clinical trials includes sorafenib that inhibits BRAF, VEGF, PDGFR,/ERK signalling and sunitinib that inhibits VEGFR, PDGFR signalling). Immunotherapy using antibodies that inhibit immune checkpoint signals within tumors are generating great interest and might mark the future of cancer immunotherapy. Three $\mathrm{mAbs}$ in this group, ipilimumab (anti CTLA-4 mAb), brolizumab and nivolumab (anti PD-1 antibody) are 
approved by the US Food and Drug Administration for the treatment of metastatic melanoma. Many pre-clinical and clinical studies are underway that test these agents in many other cancers including gastric cancer $[21,22]$, but is beyond the scope of this review.

\section{Conclusion}

Although the genetic and molecular alterations in gastric cancer present us with several logical targets, the outcomes from targeted therapies have not been very encouraging thus far. Recent studies have observed distinct molecular aberrations in gastric cancer that prevail preferentially in cancer subtypes. This may help to explain the reason behind disappointing results of many published trials in advance gastric cancer patients, where the study populations were heterogeneous regardless of clinicopathological characteristics of the primary tumor [3]. One of the major setbacks encountered in the development of targeted therapy is the lack of biomarkers that would facilitate patient stratification. To better evaluate novel targeted agents, it would be best to select small patient subsets which may benefit from molecular characterisation. More importantly, the addition of a molecular classification system to the current pathological classification system may help in selection of patient subsets who could further benefit from targeted therapies.Combinational approaches using targeted therapies and current chemotherapies regimens should also be carefully selected as it may cause toxicity concerns leading to dose reductions and inferior survival outcomes [23]. However, with growing understanding of the biology and underlying molecular heterogeneity of gastric cancer, efforts are surely underway in further improving the scope and efficacy of antibody mediated molecular targeting in gastric cancer.

\section{References}

1. Shin HR, Carlos MC, Varghese C (2012) Cancer control in the Asia Pacific region: current status and concerns. Jpn J Clin Oncol 42: 867-881. [Crossref]

2. Biondi A, Persiani R, Cananzi F, Zoccali M, Vigorita V, et al. (2010) R0 resection in the treatment of gastric cancer: room for improvement. World J Gastroenterol 16 : 3358-3370. [Crossref]

3. Wong H, Yau T (2013) Molecular targeted therapies in advanced gastric cancer: does tumor histology matter? Therap Adv Gastroenterol 6: 15-31. [Crossref]

4. Scott AM, Wolchok JD, Old LJ (2012) Antibody therapy of cancer. Nat Rev Cancer 12: 278-287. [Crossref]

5. Weiner GJ (2015) Building better monoclonal antibody-based therapeutics. Nat Rev Cancer 15: 361-370. [Crossref]

6. Wang J, Reiss KA, Khatri R, Jaffee E, Laheru D (2015) Immune Therapy in GI Malignancies: A Review. J Clin Oncol 33: 1745-1753. [Crossref]

7. Schlessinger J (2000) Cell signaling by receptor tyrosine kinases. Cell 103: 211-225. [Crossref]
8. Robinson DR, Wu YM, Lin SF (2000) The protein tyrosine kinase family of the human genome. Oncogene 19: 5548-5557. [Crossref]

9. Hubbard SR, Till JH (2000) Protein tyrosine kinase structure and function. Annu Rev Biochem 69: 373-398. [Crossref]

10. Morishita A, Gong J, Masaki T (2014) Targeting receptor tyrosine kinases in gastric cancer. World J Gastroenterol 20: 4536-4545. [Crossref]

11. Yano T, Doi T, Ohtsu A, Boku N, Hashizume K, et al. (2006) Comparison of HER2 gene amplification assessed by fluorescence in situ hybridization and HER2 protein expression assessed by immunohistochemistry in gastric cancer. Oncol Rep 15: 65-71. [Crossref]

12. Koeppen HK, Wright BD, Burt AD, Quirke P, McNicol AM, et al. (2001) Overexpression of HER2/neu in solid tumours: an immunohistochemical survey. Histopathology 38: 96-104. [Crossref]

13. Bang YJ, Van Cutsem E, Feyereislova A, Chung HC, Shen L, et al. (2010) Trastuzumab in combination with chemotherapy versus chemotherapy alone for treatment of HER2positive advanced gastric or gastro-oesophageal junction cancer (ToGA): a phase 3 , open-label, randomised controlled trial. Lancet 376: 687-697. [Crossref]

14. Kim MA, Lee HS, Lee HE, Jeon YK, Yang HK, et al. (2008) EGFR in gastric carcinomas: prognostic significance of protein overexpression and high gene copy number. Histopathology 52: 738-746. [Crossref]

15. Van Cutsem E, Peeters M, Siena S, Humblet Y, Hendlisz A, et al. (2007) Open-label phase III trial of panitumumab plus best supportive care compared with best supportive care alone in patients with chemotherapy-refractory metastatic colorectal cancer. J Clin Oncol 25: 1658-1664. [Crossref]

16. Waddell T, Chau I, Cunningham D, Gonzalez D, Okines AF, et al. (2013) Epirubicin, oxaliplatin, and capecitabine with or without panitumumab for patients with previously untreated advanced oesophagogastric cancer (REAL3): a randomised, open-label phase 3 trial. Lancet Oncol 14: 481-489. [Crossref]

17. Wang X, Chen X, Fang J, Yang C (2013) Overexpression of both VEGF-A and VEGF-C in gastric cancer correlates with prognosis, and silencing of both is effective to inhibit cancer growth. Int J Clin Exp Pathol 6: 586-597. [Crossref]

18. Qiu MZ, Xu RH (2013) The progress of targeted therapy in advanced gastric cancer Biomark Res 1: 32. [Crossref]

19. Shah MA (2014) Gastrointestinal cancer: targeted therapies in gastric cancer-the dawn of a new era. Nat Rev Clin Oncol 11: 10-11. [Crossref]

20. Nakajima M, Sawada H, Yamada Y, Watanabe A, Tatsumi M, et al. (1999) The prognostic significance of amplification and overexpression of c-met and c-erb B-2 in human gastric carcinomas. Cancer 85: 1894-1902. [Crossref]

21. Ralph C, Elkord E, Burt DJ, O’Dwyer JF, Austin EB, et al. (2010) Modulation of lymphocyte regulation for cancer therapy: a phase II trial of tremelimumab in advanced gastric and esophageal adenocarcinoma. Clin Cancer Res 16: 1662-1672. [Crossref]

22. Herbst RS, Soria JC, Kowanetz M, Fine GD, Hamid O, et al. (2014) Predictive correlates of response to the anti-PD-L1 antibody MPDL3280A in cancer patients. Nature 515: 563-567. [Crossref]

23. Smyth EC, Cunningham D (2012) Targeted therapy for gastric cancer. Curr Treat Options Oncol 13: 377-389. [Crossref]

Copyright: (C) 2015 Subhash VV. This is an open-access article distributed under the terms of the Creative Commons Attribution License, which permits unrestricted use, distribution, and reproduction in any medium, provided the original author and source are credited. 